\title{
Refractory macular edema in X-linked juvenile retinoschisis
}

\author{
Rashed Mustafa Nazzal(®), Robert Rejdak(1) \\ Department of General Ophthalmology, Medical University of Lublin, Lublin, Poland
}

\begin{abstract}
X-linked juvenile retinoschisis (XLRS) is one of the most common X-linked inherited, bilateral vitreoretinal dystrophies affecting males in the first two decades of life. In this article, we present a case report of a young man who presented with gradual decrease in visual acuity in both eyes that was attributed to macular edema and foveal schisis in the inner retinal layers. He was investigated by appropriate ocular images which were consistent with a diagnosis of XLRS. He was treated by anti-vascular endothelial growth factor (anti-VEGF) intravitreal injection, dorzolamide eye drops and intravitreal steroids injection without improvement.
\end{abstract}

KEY WORDS: retinoschisis; macular edema; juvenile; carbonic anhydrase inhibitors; vascular endothelial growth factor

Ophthalmol J 2019; Vol. 4, 46-48

\section{INTRODUCTION}

$\mathrm{X}$-linked juvenile retinoschisis (XLRS) is a relatively common early-onset retinal degenerative disease that affects males early in life [1]. Characteristic features include mild to severe loss in central vision, splitting of inner retinal layers in the fovea and in peripheral retina, and a reduction in b-wave amplitude in Electroretinogram (ERG) [2]. Currently, there is no approved treatment for XLRS and management involves treating complications, which commonly include vitreous hemorrhage and retinal detachments [3].

\section{CASE REPORT}

Twenty-year-old male patient presented complaining of bilateral gradual painless decrease in vision during the last few months. He denied any nyctalopia. The patient visited another doctor who has diagnosed him with macular edema and treated him previously with bilateral anti-VEGF (ranibizumab $0.5 \mathrm{mg} / 0.05 \mathrm{cc}$ ). On examination, the pa- tient's best corrected visual acuity of the right eye was 0.2 and of the left eye was 0.3 . Anterior segments of both eyes were within normal limits and intraocular pressure was $12 \mathrm{~mm} \mathrm{Hg}$ in each eye. Fundus examination (Fig. 1) in both eyes revealed blunted foveal reflex, peripheral inferiotemporal oval retinoschisis in the form of oval defects and retinal veils. Ocular coherent tomography (OCT) was done (Fig. 2) which revealed bilateral asymmetrical foveal cystic spaces and schisis in the inner retinal layer. Fundus Fluorescein Angiography (FFA) was done (Fig. 3) and showed bilateral dry macula and peripheral hypofluorescence in the area of retinal veils. ERG test showed selective decreased $\mathrm{b}$-wave response. All of these findings are consistent with XLRS. Because the patient received the injection of anti-VEGF 2 months ago without any improvement (as demonstrated by comparing the OCT before and after the injection), a decision was made to start the patient on topical dorzolamide eye drop (2\%) 3 times a day for 1 month. The patient came back with no improvement. A trial 


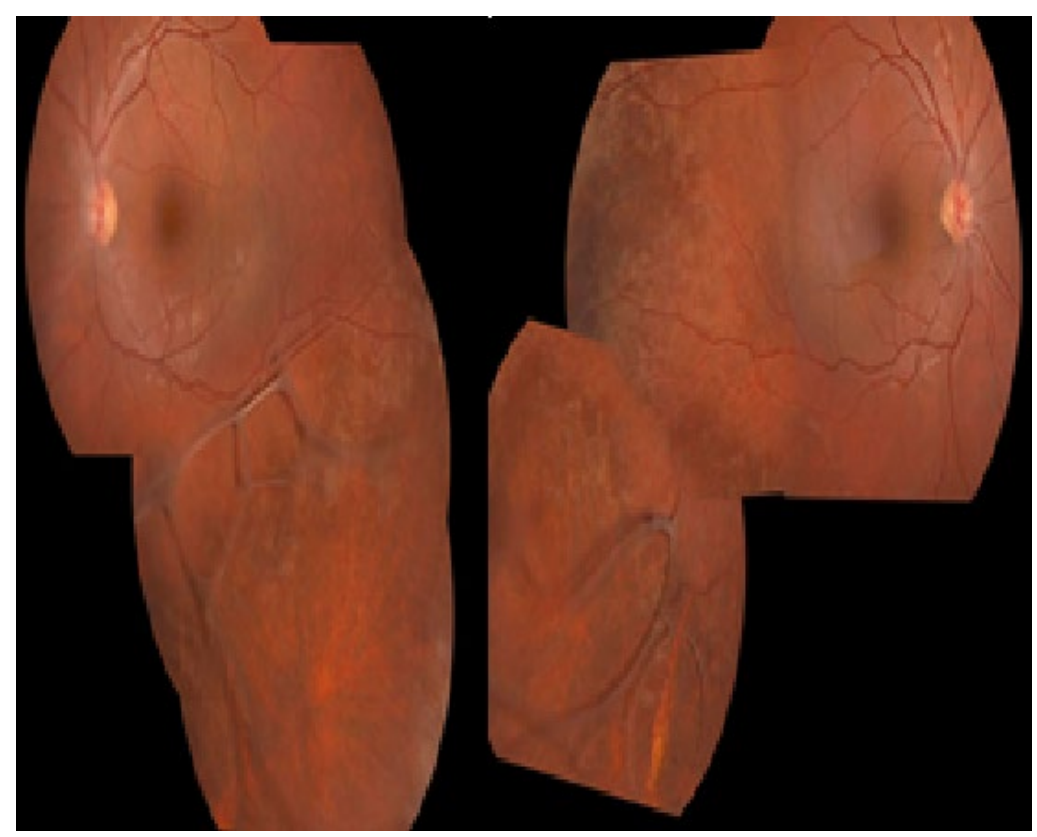

FIGURE 1. Color fundus photograph of both eyes showing the peripheral veils (left: left eye, right: right eye)

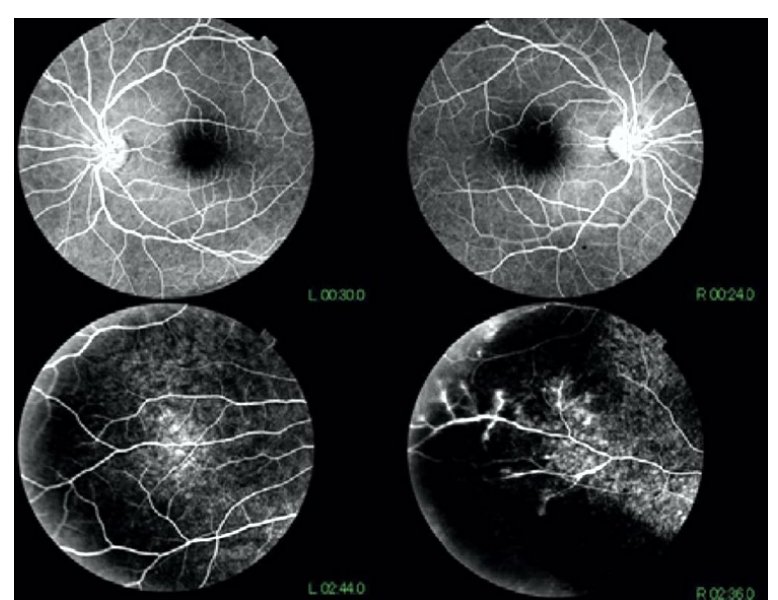

FIGURE 2. OCT of both eyes showing foveal inner layer schisis (left column: left eye, right column: right eye)

of intravitreal injection of preservative free triamcinolone $(4 \mathrm{mg} / 0.1 \mathrm{cc})$ was given and the patient was followed up for 2 months. The patient came back with no improvement also. So a decision was made just to follow up the patient.

\section{DISCUSSION}

X-linked juvenile retinoschisis is the leading cause of juvenile macular degeneration in males and leads to splitting within the inner retinal layers leading to visual deterioration [4]. Many mis-
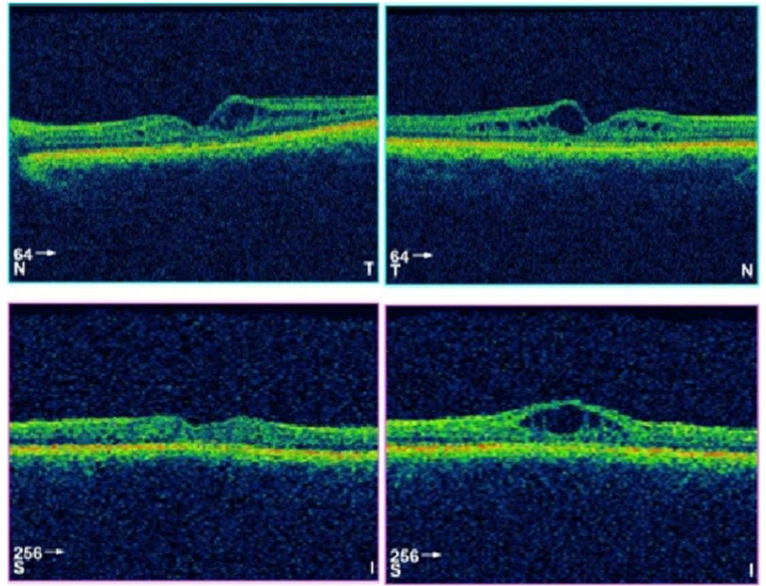

FIGURE 3. FFA for both eyes showing absence of leakage in the macula (left column: left eye, right column: right eye)

sense and protein truncating mutations have been identified in the causative retinoschisis gene (RS1) which secrets retinal protein, retinoschisin, which is implicated in cell-cell interactions and cell adhesion. Mutations cause loss of retinoschisin and consequently loss of its function [5].

The name derives from an internal splitting of the retina mostly affecting the temporal periphery of the fundus which occurs in less than $50 \%$ of affected individuals, whereas foveal involvement is present in all affected patients, which is usually associated with moderate visual loss [1]. Nevertheless, 
these figures vary according to the studied population and the mutated gene [6].

If the inner sheet of the schisis degenerates, the retinal vessels may remain running free through the vitreous cavity presenting as so called vitreous veils. If additional breaks occur in the outer sheet of the schisis, a retinal detachment may occur [1]. Vitreous hemorrhage is also a known complication resulting from the rupture of unsupported blood vessels or pre-retinal neovascularization [7].

Macular edema in this disease is peculiar because of the absence of leakage on FFA, which narrows the differential diagnosis to a few diseases, namely, some types of retinitis pigmentosa, X-linked retinoschisis, nicotinic acid maculopathy, and some cases of epiretinal membrane and vitreomacular traction syndrome [8].

One theoretical mechanism of macular edema involves intracytoplasmic edema of Muller cells, which may stem from an initial microvascular insult. This same process may occur in XLRS, where abnormal retinoschisin accumulation causes dysfunctional Muller cells [3]. Based on this theory, many attempts have been taken to treat the edema using anti-inflammatory medications; one such trial was taken successfully by injections of intravitreal triamcinolone acetate, dexamethasone implant, intravitreal fluocinolone acetonide implant [3], and even subteneon triamcinolone [9]. Others have tried carbonic anhydrase inhibitors, such as dorzolamide and acetazolamide [10-12]. In fact, carbonic anhydrase inhibitors are thought to enhance adhesion between retina and retinal pigment epithelium (RPE). XLRS patients with foveal cystic cavities unresponsive to or worsening on carbonic anhydrase inhibitors may benefit from discontinuation for up-to 6 months ("medication vacation") and later retreatment with the same agent. The ON/OFF medication regimen is believed to allow the RPE metabolic pump to partially recover and therefore facilitate the ability for a future response to treatment [13]. In our patient, both of these drugs were used without success.

Although anti-VEGF drugs may be an effective and safe way to prevent deterioration of XLRS with certain complications such as vitreous hemorrhage and exudative retinal detachment [6], it was used in this case to treat macular edema without any benefit.

So, we ended up with a case that is refractory to all known medications. We advocated observing the patient since then.

\section{CONCLUSION}

In conclusion, XLRS is a genetic disease with many ocular manifestations which in some patients were found to respond to pharmacotherapy. Patients with refractory edema may be observed closely.

\section{ACKNOWLEDGEMENTS}

None.

\section{CONFLICT OF INTERESTS}

No competing interests.

\section{REFERENCES}

1. Molday RS, Kellner U, Weber BHF. X-linked juvenile retinoschisis: clinical diagnosis, genetic analysis, and molecular mechanisms. Prog Retin Eye Res. 2012; 31(3): 195-212, doi: 10.1016/j.preteyeres.2011.12.002, indexed in Pubmed: 22245536.

2. Tantri $A$, Vrabec TR, Cu-Unjieng A, et al. X-linked retinoschisis: a clinical and molecular genetic review. Surv Ophthalmol. 2004; 49(2): 214-230, doi: 10.1016/j.survophthal.2003.12.007, indexed in Pubmed: 14998693.

3. Ansari WH, Browne AW, Singh RP. Juvenile X-linked retinoschisis responsive to intravitreal corticosteroids. Am J Ophthalmol Case Rep. 2017; 5: 48-51, doi: 10.1016/j.ajoc.2016.12.002, indexed in Pubmed: 29503947.

4. Forsius $\mathrm{H}$, Krause $\mathrm{U}$, Helve $\mathrm{J}$, et al. Visual acuity in 183 cases of $\mathrm{X}$ chromosomal retinoschisis. Can J Ophthalmol. 1973; 8(3): 385-393, indexed in Pubmed: 4742888.

5. Sikkink SK, Biswas S, Parry NRA, et al. X-linked retinoschisis: an update. J Med Genet. 2007; 44(4): 225-232, doi: 10.1136/ jmg.2006.047340, indexed in Pubmed: 17172462.

6. Hu QR, Huang $\mathrm{LZ}$, Chen $\mathrm{XL}$, et al. Genetic analysis and clinical features of X-linked retinoschisis in Chinese patients. Sci Rep. 2017; 7: 44060, doi: 10.1038/srep44060, indexed in Pubmed: 28272453.

7. Hu QR, Huang $L Z$, Chen $X L$, et al. X-Linked Retinoschisis in Juveniles: Follow-Up by Optical Coherence Tomography. Biomed Res Int. 2017; 2017: 1704623, doi: 10.1155/2017/1704623, indexed in Pubmed: 28286756.

8. Witkin A.J., Duker J.S. Young woman presents with decreasing vision. Ocul Surg News US Ed. 2008. https://www.healio.com/ophthalmology/retina-vitreous/news/print/ocular-surgery-news/\%7B120df068796b-4ef7-9ea5-0ad4335772e8\%7D/young-woman-presents-withdecreasing-vision.

9. Wang JK, Lin WC. Posterior subtenon injection of triamcinolone acetonide after phacoemulsification in a patient with X-linked retinoschisis. Optom Vis Sci. 2013; 90(10): e274-e277, doi: 10.1097/ OPX.0000000000000026, indexed in Pubmed: 23974665.

10. Deshmukh S, Gupta K, Das D, et al. Juvenile X-Linked retinoschisis: Response to topical dorzolamide therapy. TNOA J Ophthal Sci Res. 2018; 56(1): 35, doi: 10.4103/tjosr.tjosr 3518

11. Apushkin MA, Fishman GA. Use of dorzölamide for patients with $X$ linked retinoschisis. Retina. 2006; 26(7): 741-745, doi: 10.1097/01. iae.0000237081.80600.51, indexed in Pubmed: 16963845.

12. Ghajarnia $M$, Gorin MB. Acetazolamide in the treatment of X-linked retinoschisis maculopathy. Arch Ophthalmol. 2007; 125(4): 571-573, doi: 10.1001/archopht.125.4.571, indexed in Pubmed: 17420384.

13. Coussa RG, Kapusta MA. Treatment of cystic cavities in $X$-linked juvenile retinoschisis: The first sequential cross-over treatment regimen with dorzolamide. Am J Ophthalmol Case Rep. 2017; 8: 1-3, doi: 10.1016/j.ajoc.2017.07.008, indexed in Pubmed: 29260104. 